\title{
Un mot de la rédactrice
}

Je suis heureuse de vous présenter ce numéro de la RCEP, qui a deux segments thématiques, plusieurs notes sur la pratique ainsi que des comptes-rendu de livres à vous offrir. Le premier segment thématique, qui comprend un article de Catherine Fallon en français et un article de Lisa Birch et Steve Jacob en anglais, pour lequel M. Jacob a été le rédacteur invité, porte sur la démocratie et lévaluation. Le deuxième segment thématique est composé de textes issus des conférences invitées sur la réconciliation et lévaluation axée sur la culture présentées lors du Congrès national de la SCE en 2018; Nan Wehipeihana en est la rédactrice invitée. Nos conférencières et conférenciers ont généreusement transcrit et mis à jour leurs exposés pour en faire part à tous les membres de la SCE, et à d'autres évaluateurs et évaluatrices de partout dans le monde. J'aimerais profiter de l'occasion pour remercier Nicole Bowman-Farrell, Larry Bremner, Debbie Delancey, Kate McKegg et Nan Wehipeihana pour leurs contributions. En plus de ces deux segments, le présent numéro propose une importante mise à jour sur l'analyse de contribution préparée par John Mayne; un outil pour évaluer les partenariats, présenté par Angèle Bilodeau et Gillian Kranias; un inventaire des programmes canadiens de formation en évaluation réalisé par Theresa Hunter et James McDavid; et un modèle d'évaluation développé par Catherine Fréchette-Simard, Jonathan Bluteau et Isabelle Plante. Ce numéro comprend également une note sur la pratique et la recherche de Jeremy Acree au sujet des principes visant à guider les approches collaboratives dévaluation, ainsi qu'une note de Christopher Cook et ses collaborateurs sur lévaluation fondée sur le théâtre. Finalement, vous trouverez aussi deux comptes-rendu de livres. Je remercie les auteur.e.s qui continuent à nous envoyer leurs soumissions et leurs contributions et jespère que le présent numéro saura générer des réflexions enrichissantes pour tous nos lecteurs et toutes nos lectrices.

Isabelle Bourgeois Rédactrice en chef 